\title{
Intraday Empirical Analysis and Modeling of Diversified World Stock Indices
}

\author{
WOLFGANG BREYMANN ${ }^{1,3}$, LEAH KELLY ${ }^{2}$ and ECKHARD PLATEN ${ }^{2}$ \\ ${ }^{1}$ Eidgenössische Technische Hochschule Zürich, Risk Lab, CH-8092 Zürich, Switzerland \\ ${ }^{2}$ University of Technology Sydney, School of Finance \& Economics and Department of \\ Mathematical Sciences, PO Box 123, Broadway, NSW, 2007, Australia \\ ${ }^{3}$ Present address: Zurich University of Applied Science Winterthur, Institute for Data Analysis and \\ Process Design, $\mathrm{CH}-8401$ Winterthur, Switzerland
}

\begin{abstract}
This paper proposes an approach to the intraday analysis of diversified world stock accumulation indices. The growth optimal portfolio (GOP) is used as reference unit or benchmark in a continuous financial market model. Diversified portfolios, covering the world stock market, are constructed and shown to approximate the GOP, providing the basis for a range of financial applications. The normalized GOP is modeled as a time transformed square root process of dimension four. Its dynamics are empirically verified for several world stock indices. Furthermore, the evolution of the transformed time is modeled as the integral over a rapidly evolving mean-reverting market activity process with deterministic volatility. The empirical findings suggest a rather simple and robust model for a world stock index that reflects the historical evolution, by using only a few readily observable parameters.
\end{abstract}

Key words: world stock index, intraday analysis, growth optimal portfolio, diversified portfolio, market activity, deseasonalization, square root process

Mathematics Subject Classification: (1991) primary 90A12; secondary 60G30, 62P20

JEL Classification: G10, G13

\section{Introduction}

The purpose of this paper is the empirical construction of an intraday world stock index (WSI) that provides a good approximation of the growth optimal portfolio (GOP), see Kelly (1956) and Long (1990). It is the portfolio that maximizes logarithmic expected utility of terminal wealth. For practical applications, which include portfolio optimization and numeraire based derivative pricing, it is useful to be able to construct approximations of the GOP from observed data. This can be achieved by considering diversified portfolios, (see Platen, 2004, 2006). It is therefore the objective of this paper to study such proxies and model the intraday dynamic properties. More specifically, we will (i) construct several GOP proxies with five minutes data, (ii) compare their empirical quantities with theoretical model predictions, and (iii) try to select one on the basis of theoretical requirements. Of interest is whether the study will provide support for the theoretical findings in Platen (2006). 
Increasingly, diffusion processes are used to model financial markets in continuous time, see Merton (1992). The well-known Black-Scholes (BS) framework, in which volatility is a central concept, continues to provide the basis for quantitative financial techniques in practice. The simplest form of the BS framework implies Gaussian log-return distributions. The deviation of observed log-returns from a Gaussian distribution is an increasingly established stylized empirical fact. This is particularly apparent in the intraday regime and has been widely documented, see, for example, Dacorogna et al. (2001).

In the discrete time setting, the most advanced time-series models are able to reflect the main stylized empirical features reasonably well. For a review using ARCH-type models, see for example Bollerslev et al. (1992). In general, however, the time-aggregation properties of these models make it difficult to integrate intraday information into a typically daily discrete time framework. The unsatisfactory behavior of the coefficients of a GARCH $(1,1)$ model under time aggregation is demonstrated in Dacorogna et al. (2001). Continuous time diffusion type models, on the other hand, can resolve much of this problem. Furthermore, continuous time models benefit from the high observation frequency realized with intraday data, in particular, for the estimation of the diffusion coefficients.

The benchmark approach (BA), see Platen $(2004,2006)$, uses the GOP as a central building block and generalizes existing financial market modeling approaches. By using the BA, a GOP model is obtained that is able to accommodate many of the known empirical feature of financial markets. The practical availability of a suitable proxy for the GOP will be demonstrated. This allows a number of applications of the BA with regards to portfolio optimization, derivative pricing, integrated risk management and the efficient use of intraday data.

Diffusion processes are determined by the form of their drift and diffusion coefficients. It is known that the drift coefficient of the discounted GOP is fully determined by its diffusion coefficient, see Long (1990). Indeed, the risk premium of the GOP simply equals its squared volatility. A similar property can be shown for any other security under the BA, see Platen $(2004,2006)$. The BA provides an extension to previous approaches insofar as it excludes arbitrage without requiring the existence of an equivalent risk neutral martingale measure. As shown in Platen (2002), the ideal dynamics of the GOP implies Student $t$ distributed log-returns with degrees of freedom four. This is consistent with empirical studies that indicate tail indices around four, see Hurst and Platen (1997), Müller et al. (1998) and Breymann et al. (2003).

In general, reliable estimation of the drift coefficient or trend using the relatively short period of historical financial data available is extremely difficult for financial market models. However, the diffusion coefficient is relatively simple to determine from short observation periods. In fact, inference about the diffusion coefficient benefits from the use of intraday data, allowing considerable precision. Therefore, a market model that is fully determined by the diffusion coefficient, as provided by the $\mathrm{BA}$, is highly desirable since it allows the information about the 
drift to be automatically extracted. Analysis of high-frequency data requires special techniques, see Dacorogna et al. (2001) and Goodhart and O'Hara (1997). This includes the appropriate treatment of daily and weekly seasonal patterns apparent, see Breymann et al. (2003). Thus, daily and weekly seasonal patterns need to be integrated in the modeling. We will demonstrate that this can be done in a consistent and robust manner. It leads to a reliable observation and estimation of market activity, which under the BA turns out to be a more suitable financial quantity for incorporating stylized empirical features than is volatility in the BS framework.

Initially, the analysis is illustrated using the Morgan Stanley Capital Growth World Index (MSCI). It is shown that the MSCI, despite being observed daily, approximates the GOP reasonably well. Deviations exist although these can be attributed to two shortcomings. Firstly, the lack of intraday information and secondly, the missing data from emerging markets that are not sufficiently accounted for in the MSCI. Therefore, to rectify these problems we construct three new high-frequency WSIs that are observed at five minute intervals. These appear to be more diversified than the MSCI and approximate the GOP well.

Section 2 discusses the empirical features of the MSCI and the construction of the high-frequency WSIs. Section 3 summarizes the BA for an intraday GOP and Section 4 discusses the empirical analysis and models market activity of the WSIs.

\section{World Stock Indices}

\subsection{A PRELIMINARY ANALYSIS OF A DAILY WORLD STOCK INDEX}

Initially we examine the empirical features of the daily observed Morgan Stanley Capital Growth World Index (MSCI), denominated in USD, and denoted by $V^{(\mathrm{MSCI})}(t)$. This is one of the most diversified, readily available accumulation indices. Since we are not interested in the interest rate dynamics we will study the discounted MSCI

$$
\bar{V}^{(\mathrm{MSCl})}(t)=V^{(\mathrm{MSCI})}(t) \exp \left\{-\int_{0}^{t} r(u) d u\right\}
$$

for $t \in[0, T]$. Here $r(t)$ is the short term interest rate for the USD market at time $t$.

The MSCI index includes stocks from 22 countries and is based on approximately 1200 stocks. Figure 1 shows the evolution of the discounted MSCI index over the period from January 1970 until January 2003.

The discounted index displayed in Figure 1 appears, on average, to grow exponentially. To compensate for this we introduce the normalization function

$$
\bar{\alpha}(t)=\xi \exp \{\eta t\},
$$

for $t \in[0, T]$, where $\xi>0$ is a normalization constant and $\eta>0$ is called the net growth rate of the market. 


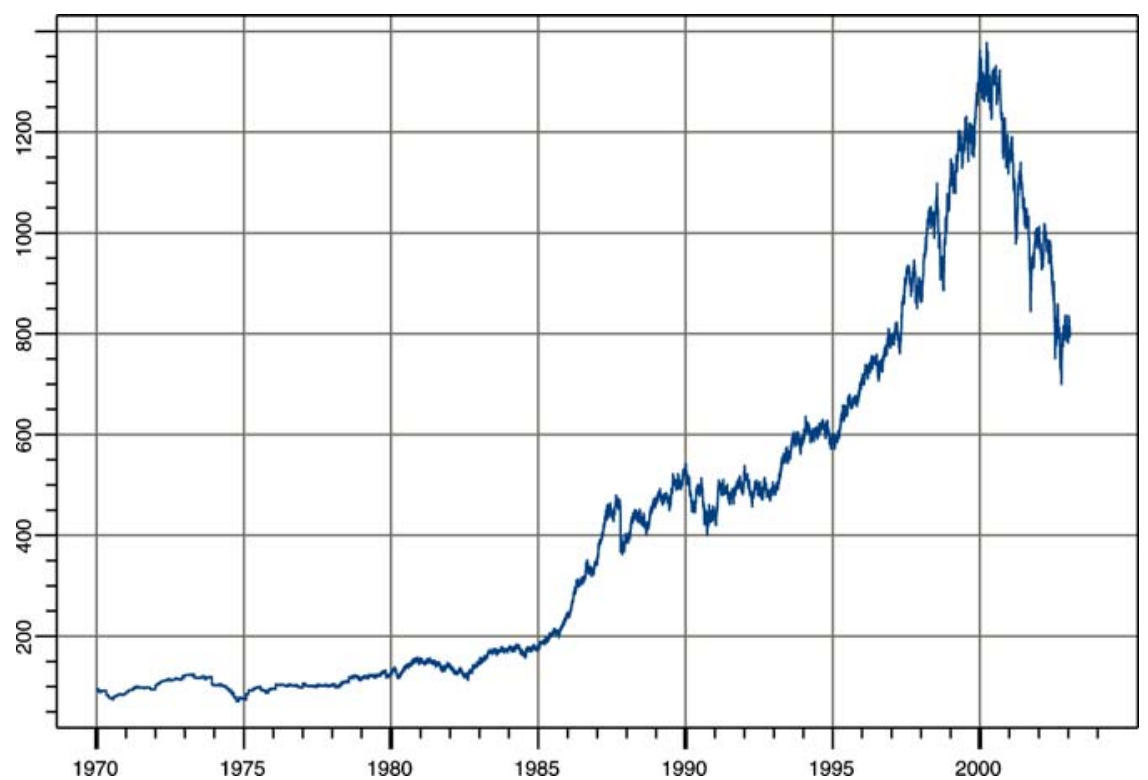

Figure 1. The discounted MSCI World Index 1970-2003.

An empirical study by Dimson et al. (2002) suggests that over the last one hundred years a discounted World Stock Index (WSI), denominated in USD, has experienced an average rate of net growth of approximately $\eta=4.8 \%$. This is consistent with the parameter range that other earlier studies detected. Thus, we introduce the normalized MSCI

$$
Y^{(\mathrm{MSCl})}(t)=\frac{\bar{V}^{(\mathrm{MSCI})}(t)}{\bar{\alpha}(t)}
$$

for $t \in[0, T]$. In the following illustrations, we have chosen $\eta=0.048$ and $\xi=10.5$, as in Platen (2004), which matches the long term growth of a market capitalization weighted WSI, as will be shown in Section 2.2. Figure 2 displays the resulting normalized MSCI over the period of thirty two years. As we will see, the time series shown in Figure 2 is in essence stationary even though fluctuations on all scales are evident and the mean reversion appears to be weak. It is interesting to note that the strength of the fluctuation depends on the level of the normalized MCSI. Periods of high values correspond to periods of large fluctuations and periods of low values correspond to periods of small fluctuations.

An important observable, when considering the fluctuations of a stochastic process $X$, is its quadratic variation $\langle X\rangle_{t}$. It is given by the limit of the sum of the squared increments of this process $X$ when the step size of the underlying time discretization tends to zero. For Brownian motion, the quadratic variation is simply the time itself. Typically, the empirically observed quadratic variation of a security 


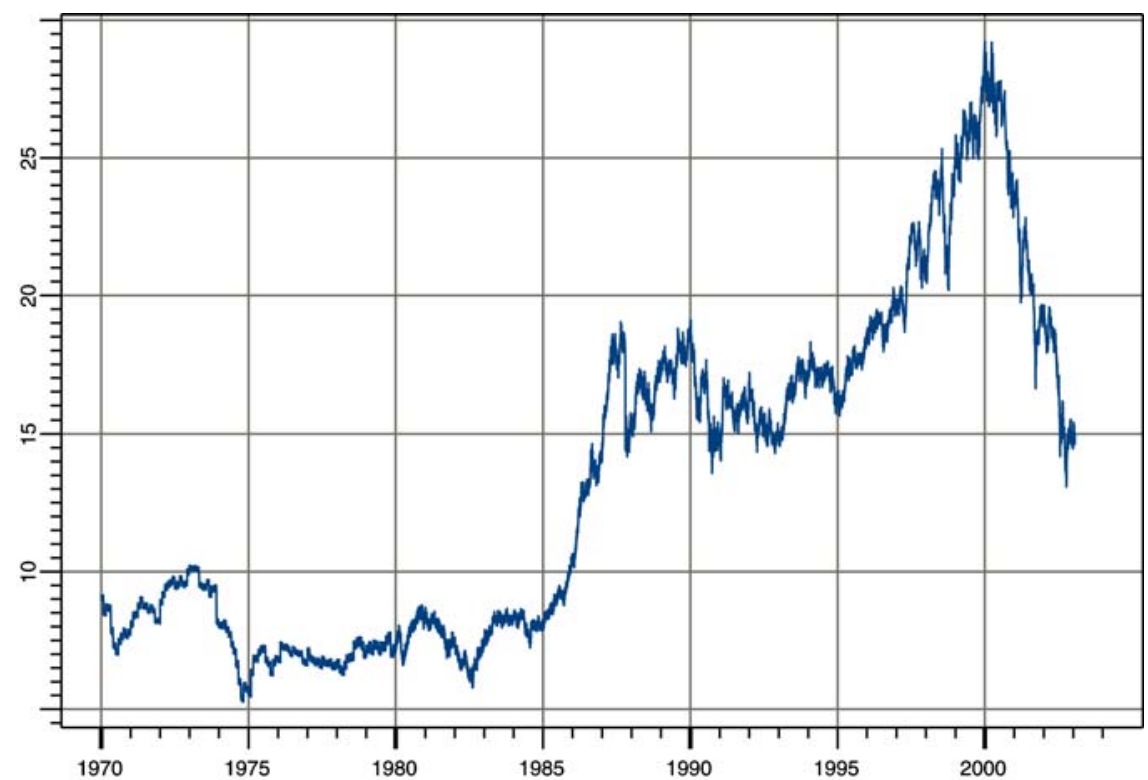

Figure 2. Normalized MSCI $Y^{(\mathrm{MSCI})}(t)$ for $1970-2003$.

or an index displays behavior more complicated than a linear function of time. The slope of quadratic variation reflects phenomena like stochastic volatility. Ideally, one would like to transform the time series of the normalized MSCI in a way that results in a process with approximately level independent fluctuations. This means that the corresponding quadratic variation should be approximately proportional to time, which implies that the diffusion term of the transformed time series would then be proportional to a Brownian motion.

With this goal in mind we consider the square root $\sqrt{Y^{(\mathrm{MSCl})}(t)}$. This particular transformation is motivated by theoretical arguments which will be presented in Section 3. Taking the square root increases fluctuations when the level of the normalized index is low and decreases fluctuations when its level is high. According to Figures 3 and 4 , the fluctuations of $\sqrt{Y^{(\mathrm{MSCI})}(t)}$ seem to be reasonably similar for high and low levels. To verify this impression Figure 5 shows the corresponding quadratic variation $\left\langle\sqrt{Y^{(\mathrm{MSCl})}}\right\rangle_{t}$, which indeed appears to be approximately proportional to time. Note that we observe in Figure 5 a slope of approximately $\frac{1}{4}$ per year. This empirical feature will be explained in Section 3.

\subsection{CONSTRUCTION OF INTRADAY WORLD STOCK INDICES}

To our knowledge there is no readily available high-frequency WSI that one could use for an intraday analysis that extends over several years. To allow for comparison, we construct three different intraday WSIs, for which we will compare key features of their dynamics. Each WSI is constructed as a self-financing portfolio consisting 


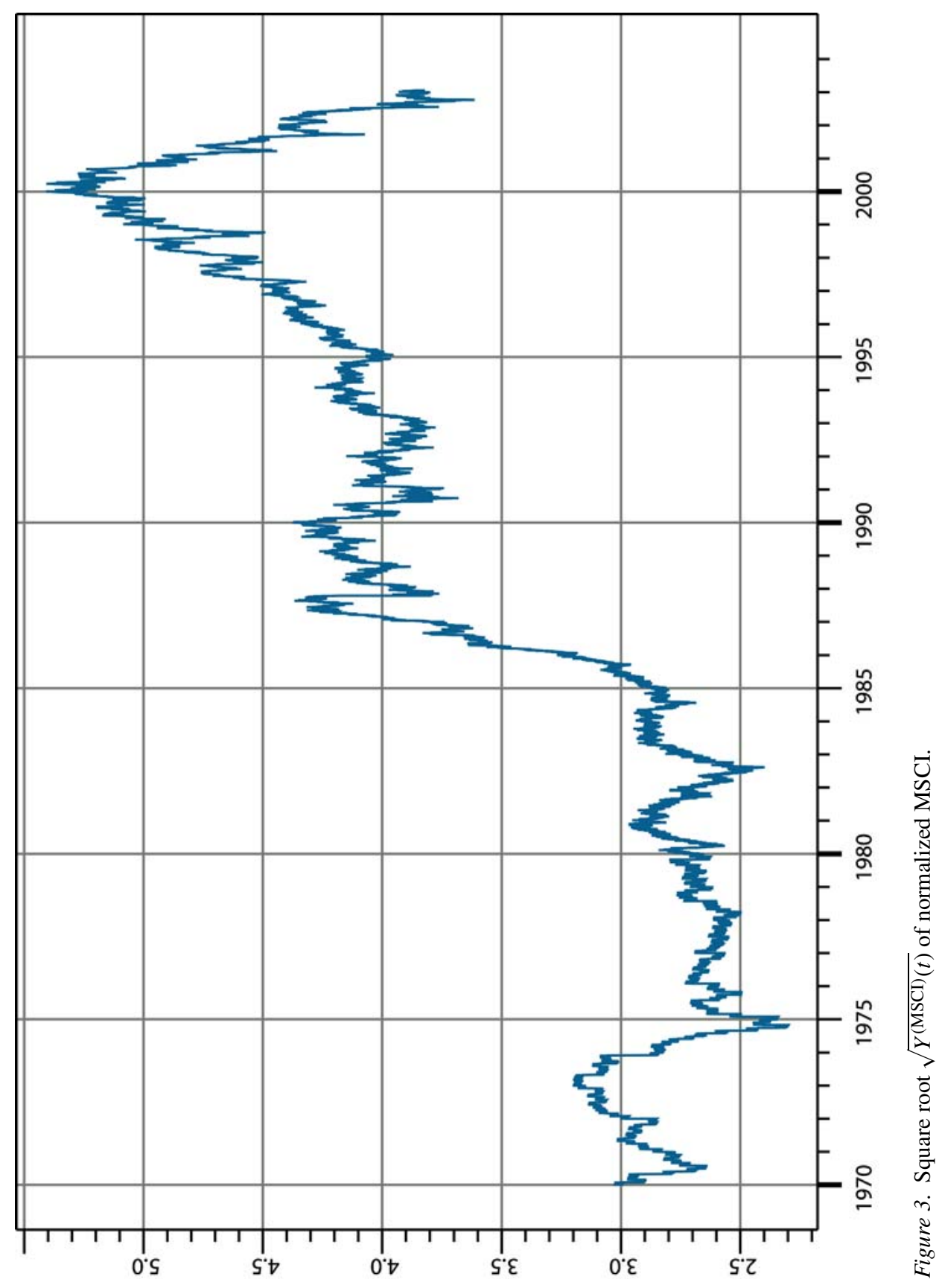




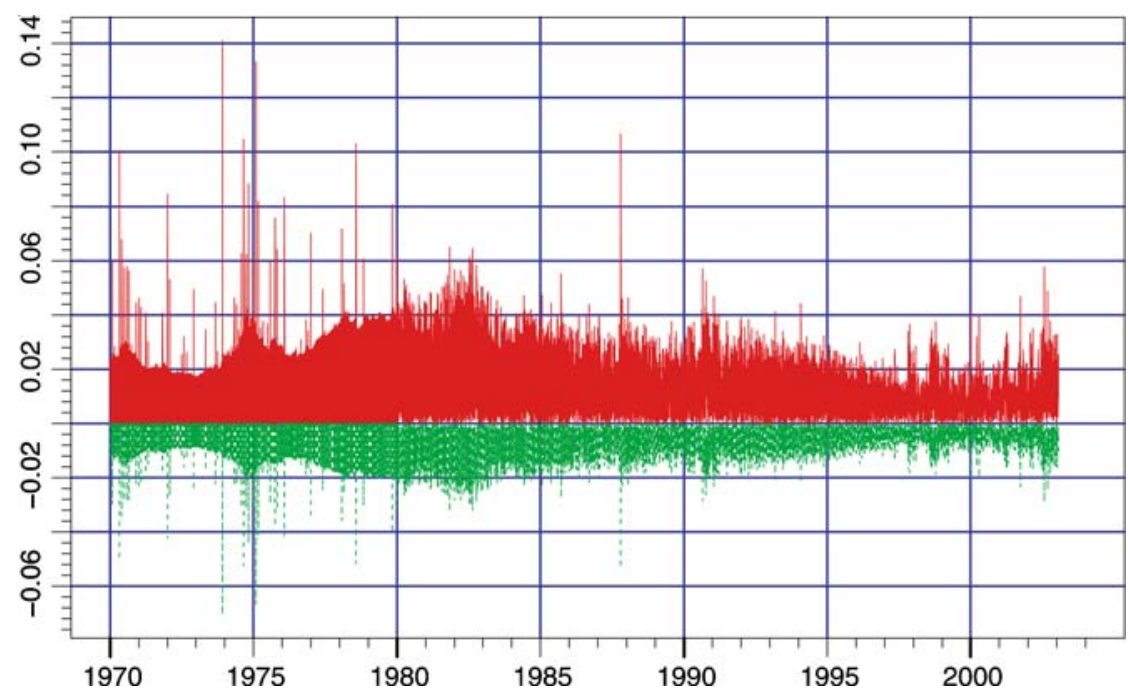

Figure 4. Absolute returns of normalized MSCI $Y^{(\mathrm{MSCI})}(t)$ (above) and $\sqrt{Y^{(\mathrm{MSCI})}(t)}$ (below).

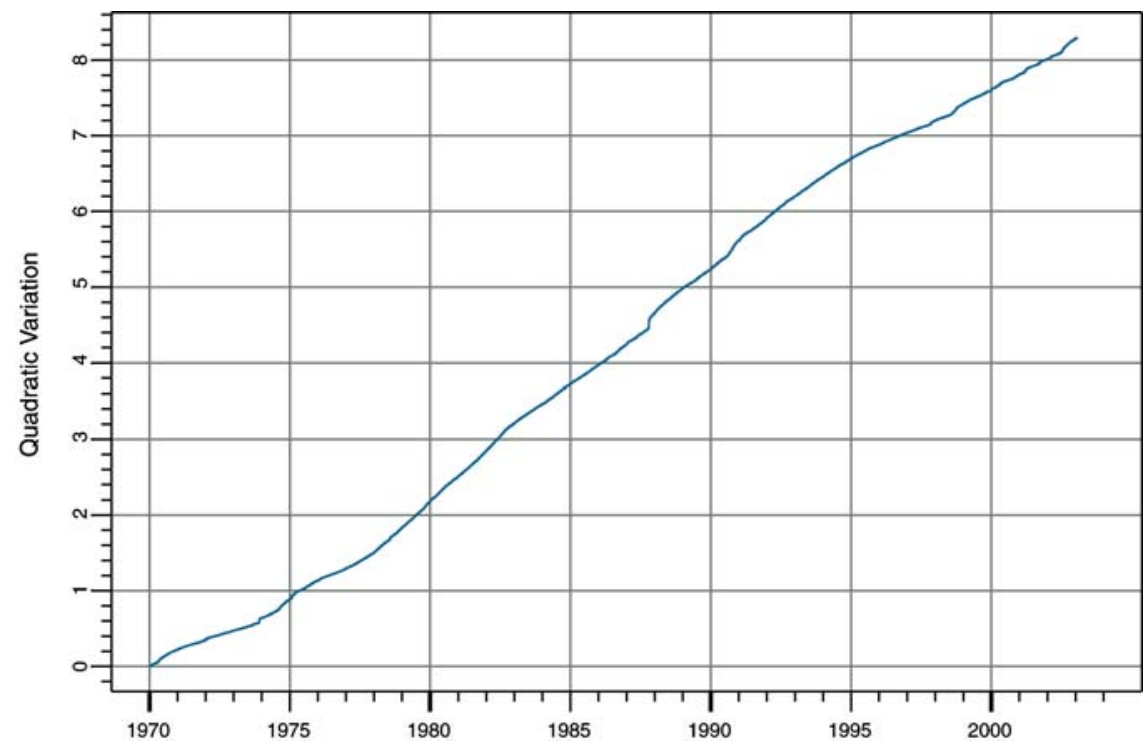

Figure 5. Quadratic variation $\left\langle\sqrt{Y^{(\mathrm{MSCI})}}\right\rangle_{t}$.

of local stock market indices taken from almost all financial markets throughout the world. The WSIs, which will be denominated in USD, are constructed from $d=34$ local stock market accumulation indices. Many of the local indices used are spot price indices. We denote by $P^{(j, i)}(t)$ the local stock spot price index at time $t$ of stock market $j \in\{1,2, \ldots, d\}$, when denominated in currency $i \in\{\mathrm{USD}, \mathrm{CHF}, \ldots\}$. 
As a first step we transform the original local spot price index into an accumulation index $I^{(j, i)}(t)$ by forming the expression

$$
I^{(j, i)}(t)=P^{(j, i)}(t) \exp \left\{\int_{0}^{t} y^{(j)}(u) d u\right\}
$$

for $t \in[0, T], j \in\{1,2, \ldots, d\}$ and $i \in\{\mathrm{USD}, \mathrm{CHF}, \ldots\}$. Here $y^{(j)}(t)$ is the continuously compounding dividend rate for the $j$ th local stock index at time $t$. Secondly, in order to form a WSI in USD, all local accumulation indices must be denominated in USD. The corresponding foreign exchange rate $X^{(i, \mathrm{USD})}(t)$ is the spot price of one US Dollar at time $t$ when measured in units of the $i$ th currency. Thus, the $j$ th local accumulation index denominated in USD is given by

$$
I^{(j, \mathrm{USD})}(t)=I^{(j, i)}(t) X^{(i, \mathrm{USD})}(t)
$$

for $t \in[0, T]$. We then obtain a WSI at time $t$, denoted by $V^{(\mathrm{WSI})}(t)$, which is denominated in USD, by forming the portfolio

$$
V^{(\mathrm{WSI})}(t)=\sum_{j=1}^{d} \delta_{(\mathrm{WSI})}^{(j)}(t) I^{(j, \mathrm{USD})}(t) .
$$

Here $\delta_{(\mathrm{WSI})}^{(j)}(t)$ denotes the number of units of the $j$ th local accumulation index held in the WSI at time $t \in[0, T]$. The value used for $\delta_{(\mathrm{WSI})}^{(j)}(t)$ corresponds to the $j$ th weight or proportion $\pi_{(\mathrm{WSI})}^{(j)}(t)$. Throughout our analysis we separate the USD interest rate evolution from our study by considering the discounted WSI $\bar{V}^{(\mathrm{WSI})}(t)$ at time $t$, which is computed from $V^{(\mathrm{WSI})}(t)$ in the same manner as shown in (2.1).

To have a small range of accumulation indices to study we construct three WSIs: the approximately Equal Weighted Index (EWI), the Market Capitalization Weighted Index (MCI) and the Gross Domestic Product Weighted Index (GDPI). The values $\delta_{(\mathrm{WSI})}^{(j)}(t)$ for each WSI are adjusted such that the proportions $\pi_{(\mathrm{WSI})}^{(j)}(t)$ for the MCI and GDPI correspond to the respective weights of the $j$ th local accumulation index, $j \in\{1,2, \ldots, d\}$. Despite its name, the EWI is not equally weighted for all markets. Developed markets were given a weight of 0.0357 and emerging markets only a weight of 0.0179 . The markets were classified as either developed or emerging to be consistent with the classification used by Morgan Stanley Capital International. The stock markets considered in the MCI account for more than 95\% of the total world market capitalization, while the GDPI weights account for more than $85 \%$ of the total world GDP. In each of the WSIs, the number of units of each of the accumulation indices is kept piecewise constant. The WSIs are rebalanced after one year has elapsed or when a local stock index is to be added to the WSI, whichever occurs first. The rebalancing dates are 05/04/1996, 17/12/1996, 
31/01/1997, 30/01/1998, 20/10/1998, 20/10/1999 and 20/10/2000. Table III at the end of this paper shows a list of the weights of the local stock indices.

To ensure that the WSIs are comparable, each index must have the same initial value. Additionally, to enable the convenient comparison of the WSIs to a readily available market index, each WSI is rescaled to have the same initial value as the MSCI at the starting date $t_{0}=$ 05/04/1996 00:00:00 Greenwich Mean Time (GMT) of our sample.

As any single local accumulation index represents a portfolio consisting of a cross section of the stocks in that local market, a WSI can be regarded as a diversified portfolio containing the stocks of all local stock markets considered. To include as many stocks as possible in the WSIs constructed, all local accumulation indices for which we could obtain high-frequency data were included. As a result of this, the three WSIs are based on between two and three thousand stocks.

In addition to local stock indices we rely on high-frequency FX spot data to transform the value of the different local stock indices into USD. The high-frequency index and FX data consists of tick-by-tick data, which was collected and filtered by Olsen Data. The period explored is from 4 April 1996 until 29 June 2001. Intraday data for a number of local stock indices start at later dates and are included into the WSIs as soon as they became continuously available. Both the original highfrequency local stock indices and FX time series are irregularly spaced. Previous tick interpolation was used to transform the observed data to regularly spaced time series with an observation time of five minutes. Furthermore, in order to form the discounted accumulated WSIs we used daily dividend rates and USD interest rates. Omitting the high-frequency information in dividends and short rates is justified because only the exponentials of integrals of these quantities, but not the values themselves, enter the formulae for the relevant quantities in our construction of the WSIs, see (2.1) and (2.4).

Figure 6 displays the three different WSIs in addition to the daily observed MSCI. It is striking that, in spite of significant differences in the weights, all WSIs appear to be very similar. In particular, the similar fluctuations of all four indices are apparent. Using the normalization function $\bar{\alpha}(t)$, given in (2.2), enables us to calculate the normalized WSI

$$
Y(t)=\frac{\bar{V}^{(\mathrm{WSI})}(t)}{\bar{\alpha}(t)}
$$

for $t \in[0, T]$, as in (2.3) .

We omit a plot of the normalized WSIs, however the normalized MCI is shown in Figure 15 in an alternative time scale. The normalized WSIs all display similar dynamics. In Platen (2004) the diversification of the WSI is shown to give a theoretical argument for this stylized fact. The hourly log-returns of the high-frequency MCI are shown in Figure 7, those of the EWI and GDPI are very similar. On the basis of their similar visual appearance it is reasonable to expect that the statistical 


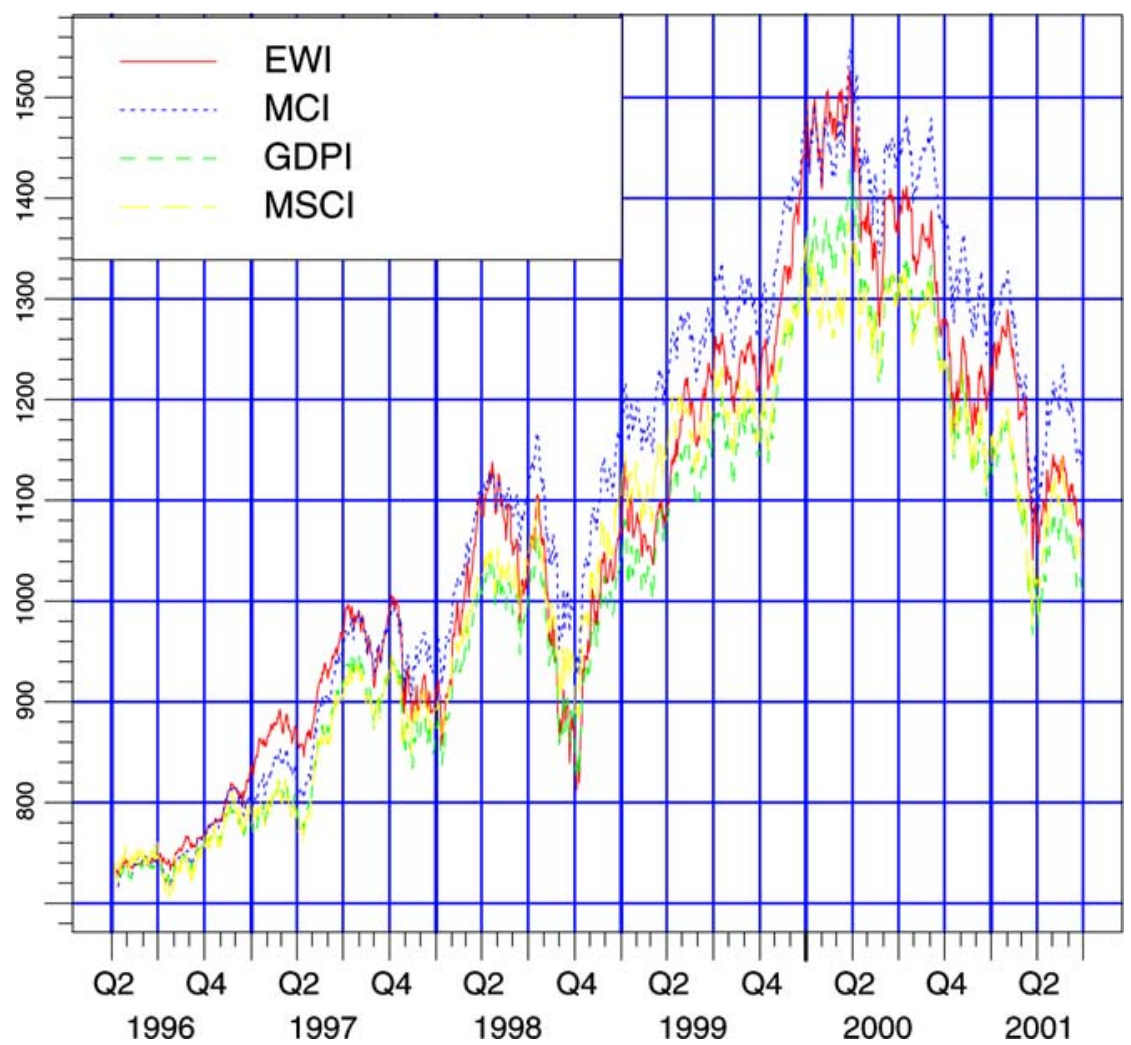

Figure 6. World Stock indices with equal weights (EWI), market capitalization adjusted weights (MCI), GDP adjusted weights (GDPI) and MSCI, where initial values are matched to the MSCI.

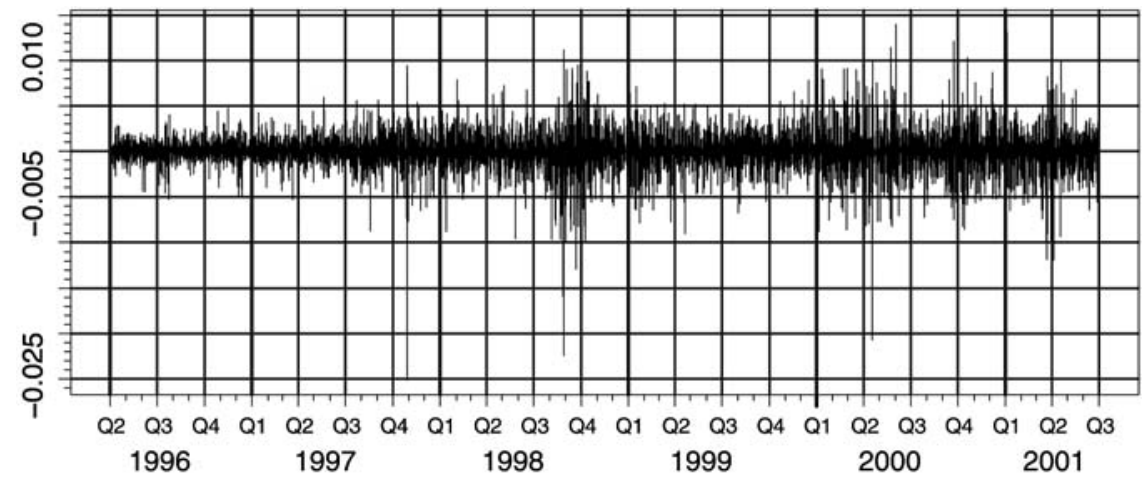

Figure 7. Hourly log-returns of the MCI. 
characteristics of the intraday WSIs will be similar as well. However, this is not obvious and needs to be explored. To place this type of analysis in a theoretically sound context, the benchmark approach (BA) is introduced in the next section.

\section{Benchmark Approach}

In the following we summarize essential facts about the BA in a somewhat informal way. To ensure the readability of this section, mathematical technicalities are omitted. For more detailed information we refer to Platen (2004).

\subsection{PRIMARY SECURITY ACCOUNTS AND PORTFOLIOS}

We consider a continuous financial market with $d+1$ primary security accounts with values $S^{(0)}(t), \ldots, S^{(d)}(t)$ at time $t \in[0, T]$, where $T$ is finite. A primary security account contains units of a given asset with all accrued income reinvested. Here $S^{(0)}(t)$ models the riskless money market savings account and $S^{(j)}(t)$, the $j$ th accumulation stock index at time $t$ as mentioned in Section 2.2. Note however that a primary security account can consist of any type of asset class. Without loss of generality, the $j$ th primary security account is supposed to satisfy the stochastic differential equation (SDE)

$$
d S^{(j)}(t)=S^{(j)}(t)\left(\left(r(t) d t+\sum_{k=1}^{d} b^{j, k}(t)\left(\theta^{k}(t) d t+d W^{k}(t)\right)\right)\right.
$$

for $t \in[0, T]$ with $S^{(j)}(0)>0, j \in\{0,1, \ldots, d\}$.

The uncertainties are modeled by $d$ independent standard Brownian motions $W^{k}=\left\{W^{k}(t), t \in[0, T]\right\}, k \in\{1,2, \ldots, d\}$ fulfilling the usual conditions, see Øksendal (1998). Only $d$ sources of uncertainty are necessary in our complete market since one of the assets is riskless. The $k$ th market price for risk $\theta^{k}(t)$ is proportional to the expected excess return that an investor obtains at time $t$ for taking risk that is modeled by the $k$ th Brownian motion.

The savings account is given by

$$
S^{(0)}(t)=\exp \left\{\int_{0}^{t} r(s) d s\right\}
$$

where $r(t)$ denotes the interest rate at time $t$. Zero volatility $b^{0, k}(t)$ applies for $S^{(0)}$ in (3.1) for all $t \in[0, T]$ and $k \in\{1,2, \ldots, d\}$. The volatility matrix $b(t)=$ $\left[b^{j, k}(t)\right]_{j, k=1}^{d}$ is assumed to be invertible to ensure that the uncertainties of the underlying securities are uniquely securitized.

Central to the BA is a portfolio that is used as benchmark and numeraire. The $j$ th component $\delta^{(j)}(t) \in(-\infty, \infty)$ of the strategy $\delta$ denotes the number of units of the 
$j$ th primary security account, which are held at time $t \in[0, T]$ in the corresponding portfolio, $j \in\{0,1, \ldots, d\}$. The value $V^{(\delta)}(t)$ of the corresponding portfolio at time $t$ can be written as the sum

$$
V^{(\delta)}(t)=\sum_{j=0}^{d} \delta^{(j)}(t) S^{(j)}(t)
$$

for $t \in[0, T]$. The portfolio is called self-financing if

$$
d V^{(\delta)}(t)=\sum_{j=0}^{d} \delta^{(j)}(t) d S^{(j)}(t)
$$

for all $t \in[0, T]$. That is, all changes in the value of the portfolio are due to gains from trade in the primary security accounts.

The self-financing portfolio value $V^{(\delta)}(t)$ satisfies according to (3.4) and (3.1) the SDE

$$
d V^{(\delta)}(t)=V^{(\delta)}(t)\left(r(t) d t+\sum_{k=1}^{d} \beta_{\delta}^{k}(t)\left(\theta^{k}(t) d t+d W^{k}(t)\right)\right)
$$

with $k$ th volatility

$$
\beta_{\delta}^{k}(t)=\sum_{j=0}^{d} \pi_{\delta}^{(j)}(t) b^{j, k}(t)
$$

and $j$ th proportion

$$
\pi_{\delta}^{(j)}(t)=\delta^{(j)}(t) \frac{S^{(j)}(t)}{V^{(\delta)}(t)}
$$

for $t \in[0, T], k \in\{1,2, \ldots, d\} \quad$ and $\quad j \in\{0,1, \ldots, d\}$.

\subsection{GROWTH OPTIMAL PORTFOLIO}

From (3.5) it follows by application of the Itô formula for the logarithm of a strictly positive portfolio $V^{(\delta)}(t)$ the SDE

$$
d \ln \left(V^{(\delta)}(t)\right)=g_{\delta}(t) d t+\sum_{k=1}^{d} \beta_{\delta}^{k}(t) d W^{k}(t)
$$


with portfolio growth rate

$$
g_{\delta}(t)=r(t)+\sum_{k=1}^{d} \beta_{\delta}^{k}(t)\left(\theta^{k}(t)-\frac{1}{2} \beta_{\delta}^{k}(t)\right)
$$

for $t \in[0, T]$.

The growth optimal portfolio (GOP), see Long (1990), is the portfolio that maximizes the portfolio growth rate (3.9). By solving the first order conditions for the corresponding quadratic maximization problem one obtains the $j$ th GOP proportion

$$
\pi_{\left(\delta_{*}\right)}^{(j)}(t)=\sum_{k=1}^{d}\left[b^{-1}(t)\right]^{k, j} \theta^{k}(t)
$$

for $t \in[0, T]$ and $j \in\{1,2, \ldots, d\}$. Here $\left[b^{-1}(t)\right]^{k, j}$ denotes the $(k, j)$ th element of the inverse $b^{-1}(t)$ of the volatility matrix. The GOP value $V^{\left(\delta_{*}\right)}(t)$ at time $t$ satisfies the SDE

$$
d V^{\left(\delta_{*}\right)}(t)=V^{\left(\delta_{*}\right)}(t)\left(r(t) d t+\sum_{k=1}^{d} \theta^{k}(t)\left(\theta^{k}(t) d t+d W^{k}(t)\right)\right)
$$

for $t \in[0, T]$. It can be seen from (3.11) that the GOP volatilities $\theta^{k}(t), k \in$ $\{1,2, \ldots, d\}$, are the corresponding market prices for risk. Consequently, the drift of the GOP can be identified via its diffusion coefficients.

To form the GOP using the proportions (3.10) is a difficult task, see for example, Long (1990). However, the GOP can be approximated in a robust manner. It is wellknown that well diversified stock portfolios behave in a similar fashion, see Figure 6. In Platen (2004) it has been shown given some weak regularity conditions, that any well diversified world stock portfolio approximates the GOP. See also Platen (2004) for a more general discussion of approximate GOPs. This makes it reasonable to assume that the WSIs constructed in Section 2.2 approximate the GOP of the world stock market. The most intuitive property of the GOP is that it systematically outperforms all other portfolios in the long run. From this perspective, the MCI in Figure 6 seems to emerge as the best approximate GOP because it outperforms the other WSIs over the observation period considered. This is consistent with the finding in Platen (2006), where the GOP equals under natural assumptions the MCI.

\subsection{DISCOUNTED GOP}

Let us discount the GOP value $V^{\left(\delta_{*}\right)}(t)$, see (3.11), at time $t$ by the savings account value $S^{(0)}(t)$, see (3.2). By application of the Itô formula together with (3.2) and 
(3.11), the discounted GOP

$$
\bar{V}^{\left(\delta_{*}\right)}(t)=\frac{V^{\left(\delta_{*}\right)}(t)}{S^{(0)}(t)}
$$

satisfies the SDE

$$
d \bar{V}^{\left(\delta_{*}\right)}(t)=\bar{V}^{\left(\delta_{*}\right)}(t)|\theta(t)|(|\theta(t)| d t+d \hat{W}(t))
$$

where

$$
d \hat{W}(t)=\frac{1}{|\theta(t)|} \sum_{k=1}^{d} \theta^{k}(t) d W^{k}(t)
$$

and the GOP volatility $|\theta(t)|$ equals the total market price for risk

$$
|\theta(t)|=\sqrt{\sum_{k=1}^{d}\left(\theta^{k}(t)\right)^{2}} .
$$

The discounted GOP drift $\alpha(t)$ is defined to be

$$
\alpha(t)=\bar{V}^{\left(\delta_{*}\right)}(t)|\theta(t)|^{2} .
$$

Allowing $\alpha(t)$ to be a, possibly stochastic, parameter process leads to a GOP volatility of the form

$$
|\theta(t)|=\sqrt{\frac{\alpha(t)}{\bar{V}^{\left(\delta_{*}\right)}(t)}} .
$$

We will demonstrate in this paper that one can model the discounted GOP drift in the form

$$
\alpha(t)=\bar{\alpha}(t) m(t)
$$

for $t \in[0, T]$, where $\bar{\alpha}(t)$ is given in (2.2). Here $m=\{m(t), t \in[0, T]\}$ denotes the nonnegative market activity process that fluctuates around one and will be specified below. This process is particularly important when modeling intraday indices as short term fluctuations in GOP volatility are captured by $m(t)$. 


\subsection{NORMALIZED GOP AND MARKET ACTIVITY}

Based on the market activity $m(t)$ introduced in the previous section the market activity time can be defined as $\psi=\{\psi(t), t \in[0, T]\}$ with

$$
\psi(t)=\int_{0}^{t} m(s) d s
$$

for $t \in[0, T]$. Note that (3.19) requires proper normalization of the market activity. It is reasonable to normalize $m(t)$ in such a way that on average the market activity time scale elapses approximately as fast as physical time. The choice in our case is such that $t=0$ years corresponds to the starting date of our sample 05/04/1996 00:00:00 GMT and $\psi(T)=5.25$ years, which is equivalent to 30/05/2001 00:00:00 GMT, the terminal date of the data available. Furthermore, we assume that

$$
\lim _{T \rightarrow \infty} \frac{1}{T} E(\psi(T))=1 .
$$

The normalized GOP $Y=\left\{Y_{\psi}, \psi \in[0, \psi(T)]\right\}$, see (2.3) and (2.7), in market activity time is defined to be

$$
Y_{\psi(t)}=Y(t)=\frac{\bar{V}^{\left(\delta_{*}\right)}(t)}{\bar{\alpha}(t)} .
$$

It is straightforward to show via the Itô formula, by using (3.13) and (3.18), that (3.21) satisfies the SDE

$$
d Y_{\psi(t)}=\frac{\bar{V}^{\left(\delta_{*}\right)}(t)}{\bar{\alpha}(t)}\left(\left(|\theta(t)|^{2}-\eta\right) d t+|\theta(t)| d \hat{W}(t)\right)
$$

and thus

$$
d Y_{\psi}=\eta\left(\frac{1}{\eta}-\frac{Y_{\psi}}{m_{\psi}}\right) d \psi+\sqrt{Y_{\psi}} d \hat{W}_{\psi}
$$

for $\psi \in[0, \psi(T)]$. Here we set

$$
d \hat{W}_{\psi(t)}=\sqrt{m(t)} d \hat{W}(t) \quad \text { and } \quad m_{\psi(t)}=m(t)
$$

for $t \in[0, T]$. The normalized GOP in market activity time in (3.22), is for $m_{\psi}=1$, a square root process with dimension four, which is a CIR process, see Cox et al. (1985). As such, the solution of (3.22) has a long term mean of $\frac{1}{\eta}$ and a speed of adjustment parameter $\eta$. One observes that the only relevant parameter in (3.22) is the net growth rate $\eta$, which is also a key parameter for the economy. If we consider 
the square root of the normalized GOP, then by (3.22) and application of the Itô formula, it evolves according to the SDE

$$
d\left(\sqrt{Y_{\psi}}\right)=\left(\frac{3}{8 \sqrt{Y_{\psi}}}-\frac{\eta \sqrt{Y_{\psi}}}{2 m_{\psi}}\right) d \psi+\frac{1}{2} d \hat{W}_{\psi}
$$

for $\psi \in[0, \psi(T)]$. It is crucial to note that the diffusion coefficient in (3.24) is constant. Therefore we obtain in market activity time the quadratic variation of $\sqrt{Y}$ in the form

$$
\langle\sqrt{Y}\rangle_{\psi}=\frac{\psi}{4}
$$

for $\psi \in[0, \psi(T)]$, see Platen (2004). Relation (3.25) holds under general circumstances since no restrictive assumptions have been imposed on the actual model dynamics. The market activity process can still be freely chosen.

Note that from the market activity time given in (3.19) and the quadratic variation of $\sqrt{Y}$ in (3.25), the market activity can be calculated as

$$
m(t)=\frac{d \psi(t)}{d t}=4 \frac{d\langle\sqrt{Y}\rangle_{t}}{d t}
$$

for $t \in[0, T]$. This implies that market activity is directly observable. A measure of the slope of the quadratic variation of the square root of the normalized GOP is all that is required. For the daily observed MSCI the quadratic variation of its square root has been shown in Figure 5. The slope of this graph is then the corresponding market activity, which remains to be modeled. However, seasonal patterns are to be expected in intraday market activity since the market experiences active and nonactive periods that depend on the time of the week, see, for example, Dacorogna et al. (2001) and Breymann et al. (2003).

\section{Intraday Market Activity}

\subsection{MARKET ACTIVITY OF THE WSIS}

As a next step we investigate the three intraday WSIs introduced in Section 2.2. According to (3.25) their market activity time is simple to calculate. In Figure 8 we show the market activity time of the MCI for the period covered by the intraday data. This allows us to calculate the market activity directly. We simply calculate the numerical derivative corresponding to (3.26) using five minute time steps. This derivative fluctuates over a wide range. Therefore, we show the logarithm $\ln (m(t))$ of this derivative in Figure 9 for the MCI over a few weeks in April/May 1996. It appears that the observed market activity process shows some seasonal patterns and reverts quickly back to a reference level. 


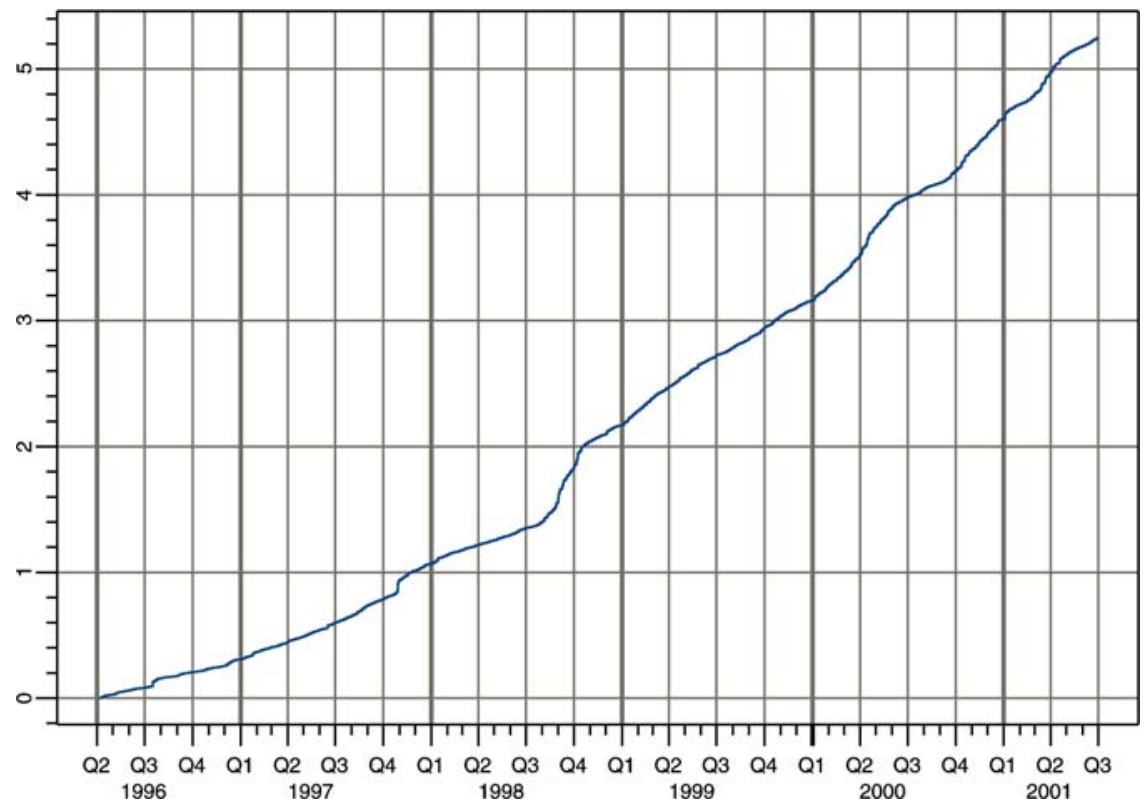

Figure 8 . The market activity time for the MCI.

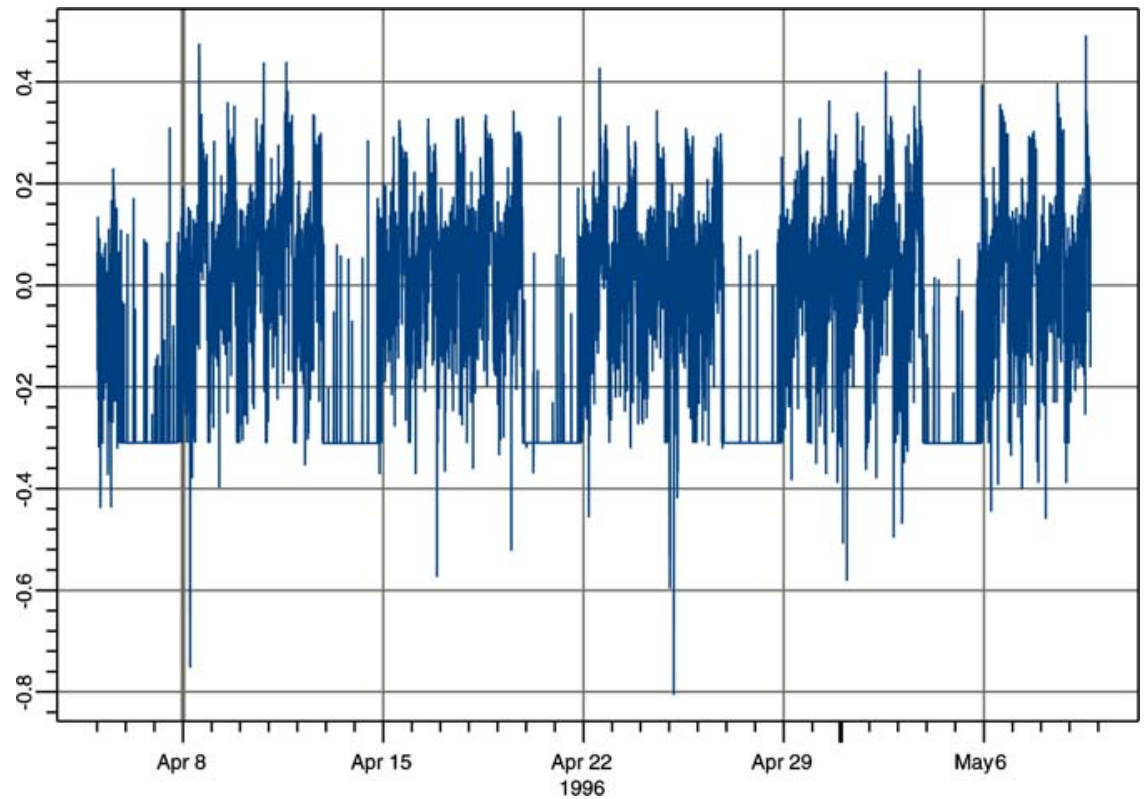

Figure 9. Logarithm of market activity, $\ln (m(t))$, for the MCI. 


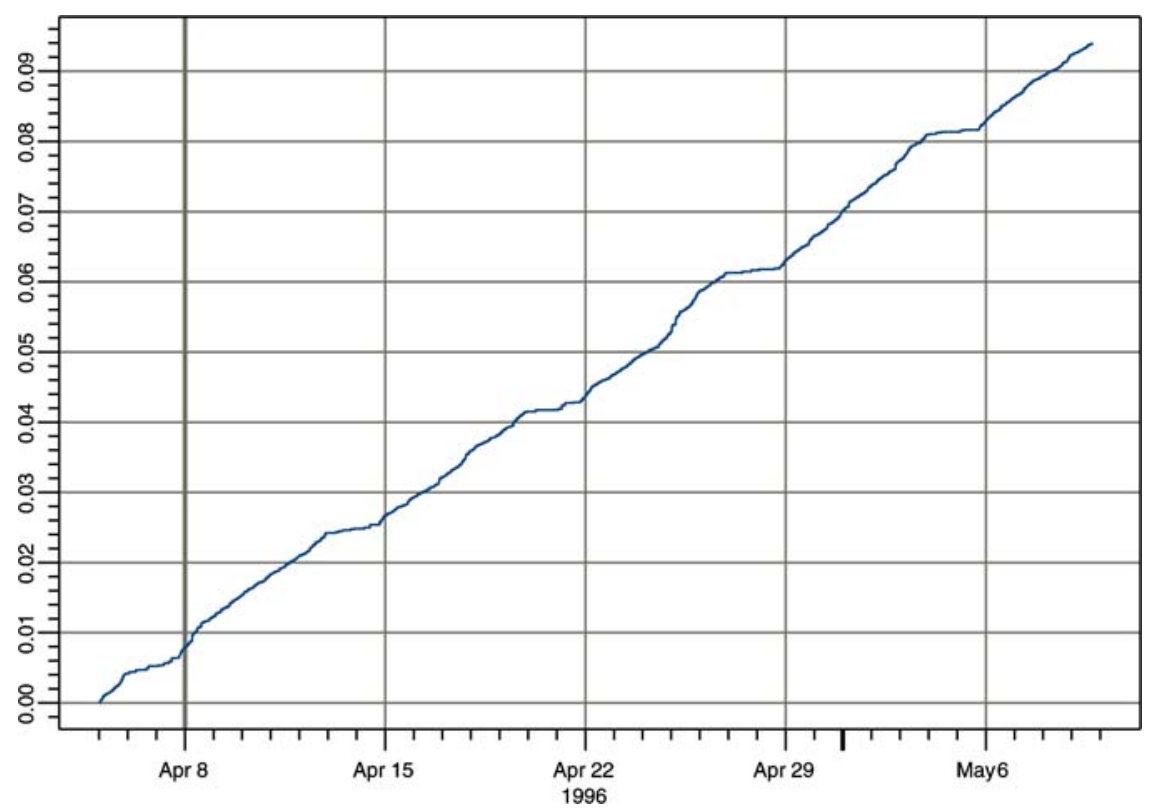

Figure 10. Quadratic variation of the logarithm of the market activity, $\langle\ln (m)\rangle_{t}$, process for the MCI.

The observed market activity processes for the EWI and GDPI look very similar and are therefore omitted. This segment of the logarithm of market activity illustrates the seasonality apparent in the average level of market activity and to some degree also some seasonality present in the average fluctuation. The presence of seasonality within the fluctuations of market activity is further confirmed by the quadratic variation $\langle\ln (m)\rangle_{t}$ of the logarithm of the market activity, which is shown for the above segment in Figure 10. Here we see that the weekends are characterized by a plateau in the quadratic variation. Notably, despite the seasonal pattern, the quadratic variation in Figure 10 appears to be almost linear for the periods when the global market is open and actively trading. Of interest is whether the seasonal patterns in the level and fluctuations of $\ln (m(t))$ can be extracted leaving only an almost stationary residual market activity process. The deseasonalization of $m(t)$ requires a two stage procedure. The first step deseasonalizes the average of the market activity and the second its diffusion coefficient.

In order to model the market activity it is necessary to characterize the relationship between its fluctuations and those of the normalized WSI. For each WSI we analyzed the covariation of the square root of the normalized WSI with the logarithm of the corresponding market. The quadratic covariation is the sum of the product of the increments of the respective processes. We observed in all three cases over the entire period that the observed covariation remains close to zero. There seems to be some evidence of a slight positive trend. However, for simplicity we 
assume that the noise that drives market activity is independent of that driving the WSI. This assumption will be relaxed in forthcoming work.

\subsection{MODELING MARKET ACTIVITY}

The expected value of the market activity is deseasonalized by the average seasonal market activity $\hat{m}(t)$ at time $t$, which is defined as the expectation

$$
\hat{m}(t)=E(m(t)) .
$$

By the law of large numbers we obtain an estimate for $\hat{m}(t)$ for each five minute interval of the week during the full observation period, 258 weeks in total, from the arithmetic average of the corresponding observed market activity. This means, we simply estimate the expectation of the market activity at time $t$ by assuming the same pattern for each week and sampling over all weeks of observation. Figure 11 displays the estimated Northern Hemisphere summertime weekly pattern of the average market activity $\hat{m}(t)$. A similar graph arises when one displays the weekly pattern for average market activity in wintertime. The average wintertime market activity turns out to be simply shifted by one hour. Note that Figure 11 is calculated from market activity, not the logarithm. The overall daily pattern is composed of several U-shaped patterns of different magnitude. The individual patterns are

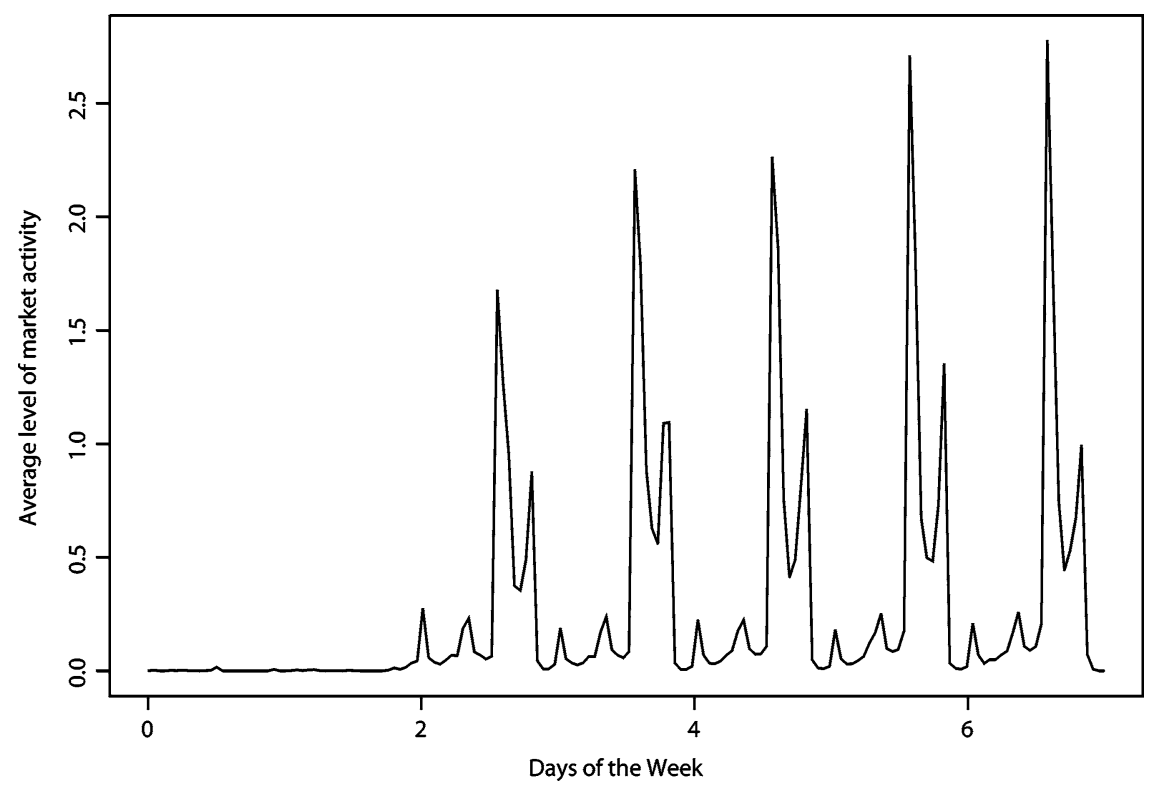

Figure 11. Weekly pattern of average market activity $\hat{m}(t)$ for summertime in the Northern Hemisphere. 
characteristic of localized, exchange-traded markets. As expected, the magnitude is highest when the European and American market are simultaneously active. It is lowest during the pacific gap, that is 21:00 till 00:00 GMT, and of course on the weekends.

Figure 10 suggests that the market activity $m(t)$ is likely to have multiplicative noise when global markets are open, with some seasonal activity volatility since the quadratic variation of its logarithm shows a piecewise linear pattern. For the open market regime, a possible model for $m(t)$ with multiplicative noise is given by the SDE

$$
d m(t)=\frac{\gamma}{2} \beta^{2}(t) m(t)\left(\frac{p(t)-1}{\gamma}-m(t)\right) d t+\beta(t) m(t) d W(t)
$$

with speed of adjustment $\gamma>0$, reference level $p(t)>0$ and activity volatility $\beta(t)>0$ for $t \in[0, T]$. The reference level $p(t)$ and the activity volatility $\beta(t)$ are assumed to exhibit some weekly periodic seasonal patterns. The specification of the drift coefficient in (4.2) is not necessary at this stage. However, this particular drift function matches the shape of the observed stationary density of the market activity that we will exploit in the following. The activity volatility $\beta(t)$ is estimated by averaging over the weekly observations of $\beta^{2}(t)$. Figure 12 shows the estimated values for $\beta(t)$ for the MCI during summertime in the Northern Hemisphere. These

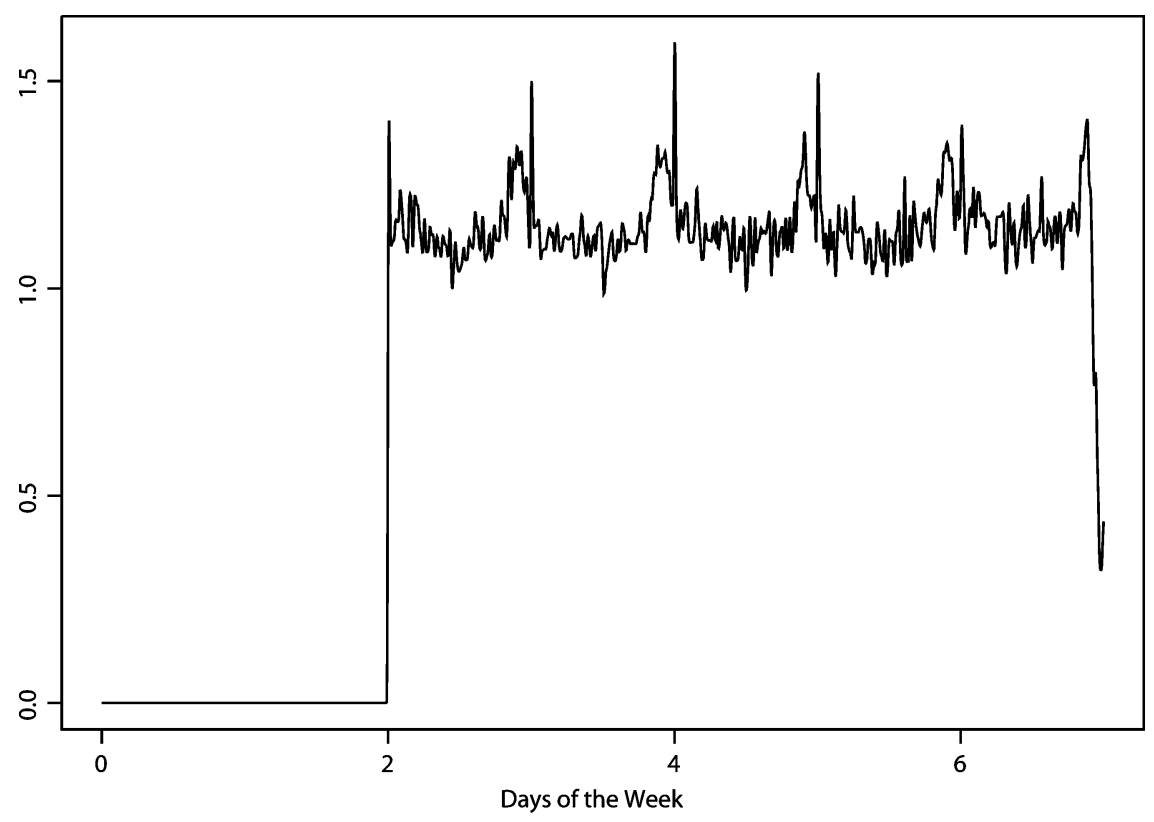

Figure 12. Average weekly pattern of activity volatility $\beta(t)$ for summertime in the Northern Hemisphere. 
estimates are also obtained from the five minute observations. Here we have set the activity volatility to zero during the weekends, since spurious observations do not allow a meaningful calculation of $\beta(t)$ outside the open market regime. Note that during the open market trading days the activity volatility is almost constant and slightly larger than one. We see that the activity volatility spikes once the Asia-Pacific markets have opened.

The activity volatility $\beta(t)$ allows us to introduce activity volatility time $\tau=$ $\{\tau(t), t \in[0, T]\}$ as

$$
\tau(t)=\langle\ln (m)\rangle_{t}=\int_{0}^{t}(\beta(u))^{2} d u
$$

for $t \in[0, T]$.

It is useful to consider the market activity in activity volatility time $\tau(t)$, denoted by $m_{\tau(t)}=m(t)$, as the presence of seasonal patterns may overshadow empirical features. Then, by (4.3) we obtain the SDE (4.2) in activity volatility time,

$$
d m_{\tau}=\frac{\gamma}{2} m_{\tau}\left(\frac{p_{\tau}-1}{\gamma}-m_{\tau}\right) d \tau+m_{\tau} d W_{\tau}
$$

where $\tau \in[0, \tau(t)], p_{\tau(t)}=p(t)$ and

$$
d W_{\tau(t)}=\beta(t) d W(t)
$$

for $t \in[0, T]$. By considering the logarithm of market activity in activity volatility time and using Itô's formula together with (4.4) we obtain the SDE

$$
d \ln \left(m_{\tau}\right)=\gamma\left(\frac{p_{\tau}}{\gamma}-m_{\tau}\right) d \tau+d W_{\tau}
$$

for $\tau \in[0, T]$. Note that the diffusion coefficient in (4.6) is constant and equal to one. Furthermore, we see that, for the open market regime, the dynamics of the logarithm of market activity are described by a mean-reverting model with speed of adjustment $\gamma$ and time dependent reference level $\frac{p_{\tau}}{\gamma}$. By considering (4.6), we may set $p_{\tau} \approx \hat{m}_{\tau} \gamma+\frac{d E\left(\ln \left(m_{\tau}\right)\right)}{d \tau}$ to account for the seasonal patterns in the reference level.

The quadratic variation of the logarithm of market activity is shown in Figure 13. It confirms the theoretical slope of one of the quadratic variation $\langle\ln (m)\rangle_{\tau}=\tau$ that follows from (4.6).

\subsection{ESTIMATION OF THE SPEED OF ADJUSTMENT}

The speed of adjustment parameter $\gamma$ in (4.4) remains to be estimated. In Figure 14 


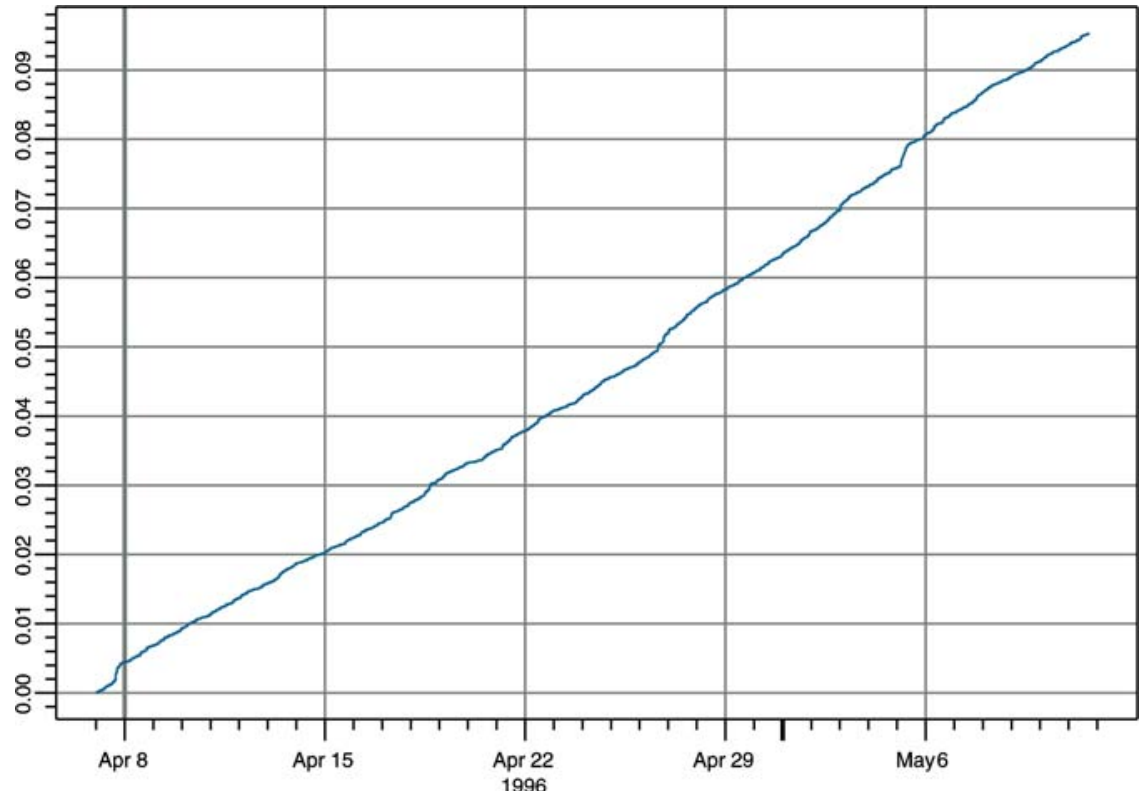

Figure 13. Quadratic variation $\langle\ln (m)\rangle_{\tau}$ of the logarithm of the normalized market activity process in activity volatility time.

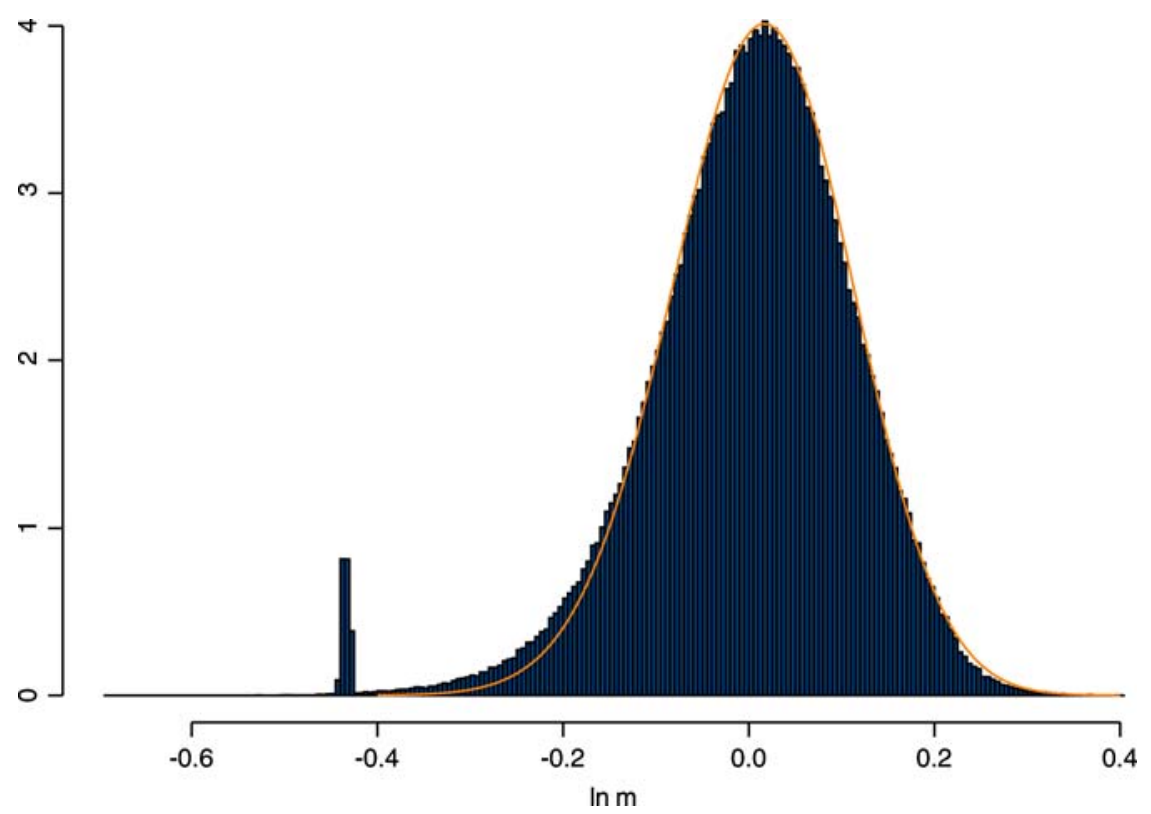

Figure 14. Histogram and estimated probability density function of $\ln \left(m_{\tau}\right)$. 
we plot the histogram of all observations of the $\operatorname{logarithm} l_{\tau}=\ln \left(m_{\tau}\right)$ of the market activity. One notes a very distinct shape of the histogram with a concentration of negative spikes around -0.4 . These negative spikes and other negative values result from the effects of market opening and closing, which can be interpreted as the typical levels of market activity in the opening and closing regimes. The given model dynamics for market activity during the open market regime, see (4.2) and (4.4), are not intended to include the opening and closing market regimes. For this reason we will ignore values less than $\underline{l}=-0.2$ in the histogram in Figure 14 for the estimation of the drift parameters for the open market regime that is modeled by (4.6). A more detailed model would need to incorporate the probability mass created in the lower tail by the superposition of the stationary densities for the different regimes.

Under the simplifying assumption that $p_{\tau}=p$ is constant, by the Fokker-Planck equation for the transition density of the solution of the SDE (4.4) the market activity process can be shown to have as stationary density a gamma density. The stationary density of the logarithm $l$ of the corresponding market activity process in activity volatility time can be written as

$$
\bar{p}_{l}(l ; \gamma, p)=\frac{(\gamma)^{p}}{\Gamma(p)} \exp \left\{-\gamma e^{l}\right\} e^{l(p-1)}
$$

Here $\Gamma(\cdot)$ is the gamma function and $\gamma$ and $p$ are the only free parameters. It is wellknown that the estimation of parameters for ergodic diffusions can be performed by fitting the observed stationary density, see Prakasa Rao (1999) and Kessler (1997). One can show for discretely observed ergodic diffusions, see, for instance, Kelly et al. (2004), that as estimators for the parameters of the stationary density one can use, for convenience, the maximum likelihood estimators for the parameters of the corresponding density of independent identically distributed observables. However, these estimators are not the most efficient ones, see Prakasa Rao (1999), as has been pointed out by an anonymous referee. A future study will investigate this problem further. In our case we perform some kind of a restricted estimation. We exclude most of the distortions caused by the effects of market opening and closing from our estimation by forming a restricted log-likelihood function

$$
L(\gamma, p)=\sum_{n=1}^{n_{T}} \mathbf{1}_{\left\{l_{\tau_{n}} \geq l\right\}} \ln \left(\bar{p}_{l}\left(l_{\tau_{n}} ; \gamma, p\right)\right)
$$

Here $n_{T}=523585$ is the total number of observations and $\mathbf{1}_{\left\{l_{\tau_{n}} \geq l\right\}}$ denotes the indicator function, which takes only observations with $l_{\tau_{n}} \geq \underline{l}$ into account, $n \in$ $\left\{1,2, \ldots, n_{T}\right\}$. A plot of the estimated probability density function of $l_{\tau}=\ln \left(m_{\tau}\right)$ based on the resulting estimate $\hat{\gamma} \approx 103$ and $\hat{p} \approx 106$ is shown in Figure 14 . We see from the effects of market opening and closing that the left tail of the histogram 
Table I. Estimates for the drift parameters of market activity in activity volatility time with $99 \%$ confidence intervals

\begin{tabular}{lll}
\hline Index & $\hat{\gamma}\left[\right.$ year $\left.^{-1}\right]$ & $\hat{p}\left[\right.$ year $\left.^{-1}\right]$ \\
\hline MCI & $103.2(89.3,116.4)$ & $105.8(92.3,119.2)$ \\
GDPI & $136.6(120.7,152.5)$ & $138.9(122.5,155.3)$ \\
EWI & $137.3(121.2,153.4)$ & $139.7(123.5,155.8)$ \\
\hline
\end{tabular}

is fatter than what is given by the theoretical gamma density. The estimates for each of the WSIs, together with $99 \%$ confidence intervals, are shown in Table I.

We point out that the assumption $p_{\tau}=p$ is not fully consistent with the seasonal patterns found in Figure 11. However, due to the small impact of the exact form of the drift function in (4.4) for values of $m_{\tau}$ near zero the differences are negligible. For extreme values of $\ln \left(m_{\tau}\right)$ the drift in (4.4) captures for constant $p_{\tau}=p$ well the modeled mean reversion and yields a stationary density that is very similar to the one shown in Figure 11. This can also be confirmed by simulation of solutions of the SDE (4.4) when using the observed seasonal average $\hat{m}_{\tau}$.

\subsection{ANALYSIS OF NORMALIZED WSIS IN MARKET ACTIVITY TIME}

In Section 3 it was shown that the normalized GOP, when observed in market activity time, see (3.19), is a square root process of dimension four, see (3.22). The trajectory of the normalized MCI in market activity time is plotted in Figure 15. By using market activity time we know that the quadratic variation of the square root of the normalized MCI should be linear with a slope close to 0.25 , see (3.25). This relationship can be confirmed by performing a simple linear regression of the quadratic variation of the square root of the normalized WSI against the corresponding observed market activity time. Note that each WSI implies its own market activity time scale, which is equivalent to four times the quadratic variation of the square root of the normalized WSI, see (3.26). For instance, the market activity time of the MCI is shown in Figure 8, where that of the GDPI and EWI are similar. The slope coefficient and $R^{2}$ value are given in Table II for all three WSIs. Of note is that the slope coefficients in Table II are not exactly 0.25 . The reason for this are

Table II. Slope coefficients and $R^{2}$ values for the quadratic variation of the square root of normalized WSIs in market activity time

\begin{tabular}{lll}
\hline Index & Slope coefficient & $R^{2}$ \\
\hline MCI & 0.245 & 0.9968 \\
GDPI & 0.232 & 0.9969 \\
EWI & 0.198 & 0.9919 \\
\hline
\end{tabular}


Table III. List of stock indices. The date indicates when the Index has been included in the World Index. In some cases large data gaps are present at the beginning of the series, and inclusion of the indices concerned is delayed. In the last columns the weights of the indices in the different versions of the World Index are displayed: (i) nearly equal weighted; (ii) weights adjusted to market capitalization; and (iii) weights adjusted to GDP

\begin{tabular}{|c|c|c|c|c|c|c|}
\hline \multicolumn{4}{|c|}{ America } & \multicolumn{3}{|c|}{ Weights } \\
\hline Country & Index & Currency & Date & (i) & (ii) & (iii) \\
\hline Argentinia & MERV & ARS & 17.12.1996 & 0.0179 & 0.0023 & 0.0102 \\
\hline Brasil & BVSP & BRL & 17.12.1996 & 0.0357 & 0.0013 & 0.004 \\
\hline $\mathrm{CA}$ & TSE300 & CAD & 05.04 .1996 & 0.0357 & 0.0251 & 0.0225 \\
\hline Mexico & IPC & MXP & 17.12.1996 & 0.0179 & 0.0016 & 0.0112 \\
\hline US & S\&P 500 & USD & 05.04 .1996 & 0.0357 & 0.4301 & 0.2859 \\
\hline \multicolumn{4}{|c|}{ Asia-Pacific } & \multicolumn{3}{|c|}{ Weights } \\
\hline Country & Index & Currency & Date & (i) & (ii) & (iii) \\
\hline Australia & AORD & AUD & 05.04 .1996 & 0.0357 & 0.0161 & 0.014 \\
\hline Hong Kong & HSI & HKD & 05.04 .1996 & 0.0357 & 0.0231 & 0.0054 \\
\hline India & BSESI & INR & 17.12.1996 & 0.0179 & 0.0201 & 0.0146 \\
\hline Indonesia & JSX & IDR & 17.12.1996 & 0.0179 & 0.0047 & 0.0081 \\
\hline Japan & Nikkei225 & JPY & 17.12.1996 & 0.0357 & 0.155 & 0.204 \\
\hline Korea & KOSPIC & KRW & 31.01 .1997 & 0.0179 & 0.0072 & 0.0195 \\
\hline Malaysia & KLSE & MYR & 17.12 .1996 & 0.0179 & 0.0158 & 0.0036 \\
\hline Philippines & PCI & PHP & 17.12.1996 & 0.0179 & 0.0041 & 0.0029 \\
\hline Singapore & STI & SGD & 05.04 .1996 & 0.0357 & 0.0079 & 0.0033 \\
\hline Taiwan & TWI & TWD & 17.12.1996 & 0.0179 & 0.0141 & 0.0102 \\
\hline Thailand & SETI & THB & 17.12.1996 & 0.0179 & 0.0049 & 0.0066 \\
\hline \multicolumn{4}{|c|}{ Europe } & \multicolumn{3}{|c|}{ Weights } \\
\hline Country & Index & Currency & Date & (i) & (ii) & (iii) \\
\hline Austria & ATX & ATS, EUR & 12.12 .1996 & 0.0357 & 0.0017 & 0.0089 \\
\hline Belgium & BEL20 & BEF, EUR & 12.12 .1996 & 0.0357 & 0.0061 & 0.0105 \\
\hline Denmark & KFX & DKK & 05.04 .1996 & 0.0357 & 0.0037 & 0.0069 \\
\hline Finland & HEX & FIM, EUR & 05.04 .1996 & 0.0357 & 0.0032 & 0.005 \\
\hline France & CAC40 & FRF, EUR & 05.04 .1996 & 0.0357 & 0.0302 & 0.0586 \\
\hline Germany & DAX & DEM, EUR & 05.04 .1996 & 0.0357 & 0.0342 & 0.0924 \\
\hline Greece & ATG & GRD, EUR & 17.12 .1996 & 0.0357 & 0.0012 & 0.0045 \\
\hline Hungary & BUXI & HUF & 31.01 .1997 & 0.0179 & 0.0023 & 0.0017 \\
\hline Ireland & ISEQ & IRP, EUR & 17.12 .1996 & 0.0357 & 0.0055 & 0.0027 \\
\hline Italy & MIB30 & ITL, EUR & 17.12.1996 & 0.0357 & 0.0132 & 0.0414 \\
\hline Netherland & AEX & NLG, EUR & 20.10 .1998 & 0.0357 & 0.0193 & 0.0153 \\
\hline
\end{tabular}

(Continued on next page) 
Table III. (Continued)

\begin{tabular}{lllllllll}
\hline \multirow{3}{*}{ Country } & Index & Currency & Date & & & & \multicolumn{3}{c}{ Weights } & & (ii) & (iii) \\
\hline Norway & OSETOT & NOK & 05.04 .1996 & & 0.0357 & 0.0029 & 0.0057 \\
Portugal & BVL30 & PTE, EUR & 17.12 .1996 & & 0.0357 & 0.0013 & 0.004 \\
Spain & IBEX & ESP, EUR & 17.12 .1996 & & 0.0357 & 0.0124 & 0.0224 \\
Sweden & SGI & SEK & 17.12 .1996 & & 0.0357 & 0.0124 & 0.0091 \\
Switzerland & SMI & CHF & 05.04 .1996 & & 0.0357 & 0.0206 & 0.0115 \\
Turkey & ICI & TRL & 31.01 .1997 & & 0.0179 & 0.0018 & 0.0068 \\
UK & FTSE ALL & GBP & 05.04 .1996 & & 0.0357 & 0.0846 & 0.0435 \\
\hline
\end{tabular}

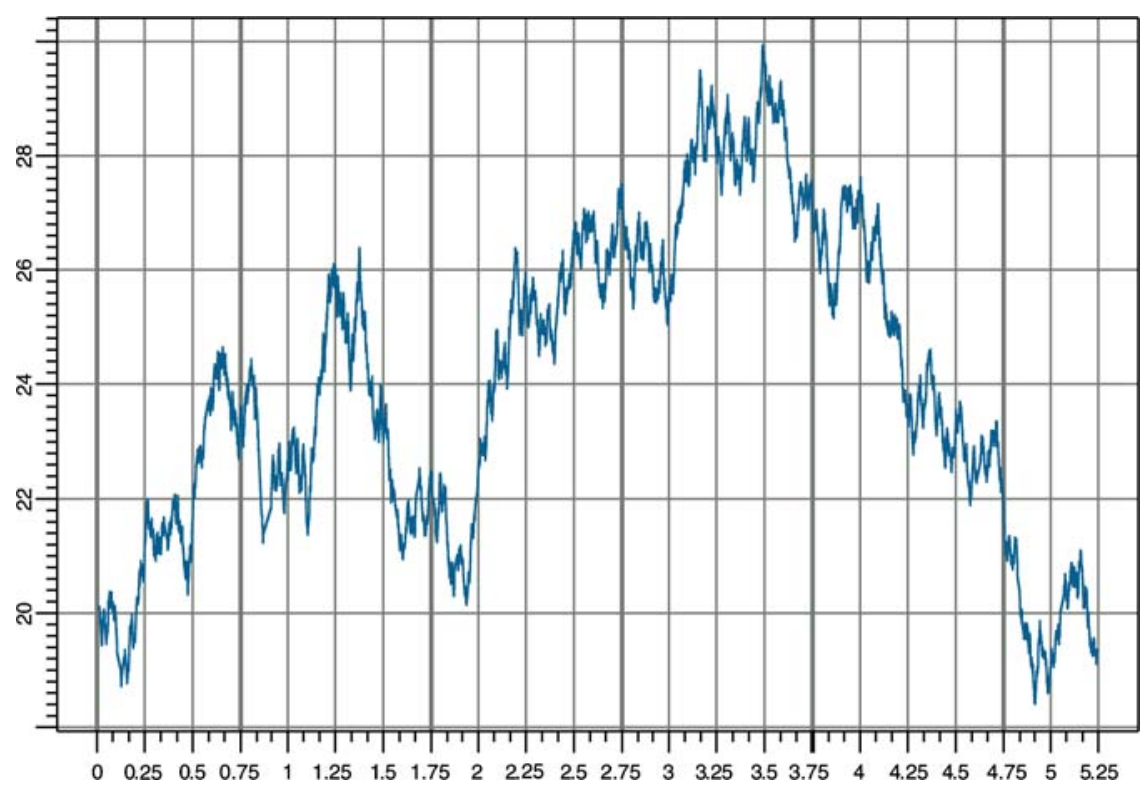

Figure 15. Normalized MCI in market activity time.

twofold. Firstly, none of these portfolios equals exactly the GOP and secondly, the net growth rate $\eta$ is not exactly constant. It should be noted that already a constant net growth rate provides a good fit. Admittedly, the regression results seem almost to be too good for a statistical analysis. However, it is the strength of the resulting model that it fits the data extremely well. Note that the MCI outperforms the EWI and the GDPI, when the quadratic variations of the square root of the corresponding normalized index are considered. However, there is not a great difference between the MCI and the GDPI. Additionally, the corresponding market activity processes in activity volatility time are shown to have the hypothesized dynamics, when the 
opening and closing regimes are omitted. Moreover, it follows from Figure 6 that the MCI has the maximum value in relation to all other indices considered, at the end of the observation period. It is a main property of the GOP that its path outperforms those of all other portfolios over long time periods. It should be emphasized that the given time period is relatively short but the fluctuations of all WSIs are extremely similar. In this respect, one may at least conclude that the above findings do not contradict a hypothesis where one considers the MCI to be the best proxy for the GOP. This provides some empirically support for the theoretical finding in Platen (2006) that in a market where all investors prefer more for less, the market portfolio equals the GOP.

\section{Conclusion}

We constructed and examined three diversified high-frequency world stock indices. A simple and robust way of calculating and modeling market activity is demonstrated. Market activity is found to contain seasonal patterns in both the drift and the diffusion term. We showed that the market activity can be modeled as a strongly mean reverting process with constant speed of adjustment incorporating the weekly periodicities contained in average market activity and activity volatility. Furthermore, we confirmed that, in market activity time, the normalized indices represent square root processes of dimension four. The world stock portfolio appears to provide a proxy for the GOP. Work is in progress on the use of the MCI for applications of the benchmark approach in derivative pricing, portfolio management and integrated risk management.

\section{Acknowledgement}

Wolfgang Breymann acknowledges support for this research by Credit Suisse Group, Swiss Re, UBS AG through RiskLab, Switzerland and the University of Technology Sydney. We thank Olsen Data for providing the high frequency data and acknowledge Thomson Financial as the provider for daily data.

\section{References}

Bollerslev, T., Chou, R. and Kroner, K. (1992) ARCH modelling in finance: A review of the theory and empirical evidence, J. Econometrics 52, 5-59.

Breymann, W., Dias, A. and Embrechts, P. (2003) Dependence structures for multivariate highfrequency data in finance, Quant. Finance 3(1), 1-14.

Cox, J. C., Ingersoll, J. E. and Ross, S. A. (1985) A theory of the term structure of interest rates. Econometrica 53, 385-407.

Dacorogna, M., Gençay, R., Müller, U., Olsen, R. and Pictet, O. (2001) An Introduction to HighFrequency Finance. Academic Press, San Diego, CA.

Dimson, E., Marsh, P. and Staunton, M. (2002) Triumph of the Optimists: 101 Years of Global Investment Returns. Princeton University Press. 
Goodhart, C. and O'Hara, M. (1997) High-frequency data in financial markets: Issues and applications. J. Empirical Finance 4, 73-114.

Hurst, S. R. and Platen, E. (1997) The marginal distributions of returns and volatility. In Y. Dodge (ed.), $L_{1}$-Statistical Procedures and Related Topics, Vol. 31 of IMS Lecture Notes - Monograph Series, Institute of Mathematical Statistics Hayward, California pp. 301-314.

Kelly, J. R. (1956) A new interpretation of information rate. Bell Syst. Techn. J. 35, 917-926.

Kelly, L., Platen, E. and Sørensen, M. (2004) Estimation for discretely observed diffusions using transform functions. In Stochastic Methods and Their Applications, Vol. 41A of J. Appl. Probab., Applied Prob. Trust, pp. 99-118.

Kessler, M. (1997) Estimation of an ergodic diffusion from discrete observations. Scand. J. Statist. 24, 211-229.

Long, J. B. (1990) The numeraire portfolio. J. Financial Economics 26, 29-69.

Merton, R. C. (1992) Continuous-Time Finance. Blackwell, Oxford.

Müller, U., Dacorogna, M. and Pictet, O. (1998) Hill, bootstrap and jackknife estimators for heavy tails. In R. Adler, R. Feldman, and M. Taqqu (eds.), A Practical Guide to Heavy Tails: Statistical Techniques for Analysing Heavy Tailed Distributions, Birkhäuser, Boston, pp. 283-310.

Øksendal, B. K. (1998) Stochastic Differential Equations. An Introduction with Applications (5th ed.). Universitext. Springer.

Platen, E. (2002) Arbitrage in continuous complete markets. Adv. Appl. Probab. 34(3), 540-558.

Platen, E. (2004) Modeling the volatility and expected value of a diversified world index. Int. J. Theor. Appl. Finance 7(4), 511-529.

Platen, E. (2006) A benchmark approach to finance Math. Finance 16(1), 131-151.

Prakasa Rao, B. L. S. (1999) Statistical Inference for Diffusion Type Processes. Arnold Publishers, Oxford University Press. 